\title{
Rapid Conversion of Mutant IDH1 from Driver to Passenger in a Model of Human Gliomagenesis
}

\author{
Tor-Christian Aase Johannessen ${ }^{1,3}$, Joydeep Mukherjee ${ }^{1}$, Pavithra Viswanath ${ }^{2}$, Shigeo \\ Ohba $^{1}$, Sabrina M. Ronen ${ }^{2}$, Rolf Bjerkvig ${ }^{3}$, and Russell O. Pieper ${ }^{1}$ \\ ${ }^{1}$ The Department of Neurological Surgery, University of California, San Francisco, San Francisco, \\ California 94158, USA \\ ${ }^{2}$ The Department of Radiology, University of California, San Francisco, San Francisco, California \\ 94158, USA \\ ${ }^{3}$ The Kristian Gerhard Jebsen Brain Tumor Research Centre, Department of Biomedicine, \\ University of Bergen, Jonas Lies Vei 91, 5009 Bergen, Norway
}

\begin{abstract}
Missense mutations in the active site of isocitrate dehydrogenase 1 (IDH1) biologically and diagnostically distinguish low-grade gliomas and secondary glioblastomas from primary glioblastomas. IDH1 mutations lead to the formation of the oncometabolite 2-hydroxyglutarate (2$\mathrm{HG}$ ) from the reduction of $a$-ketoglutarate $(a-K G)$, which in turn facilitates tumorigenesis by modifying DNA and histone methylation as well blocking differentiation processes. While mutant IDH1 expression is thought to drive the gliomagenesis process, the extent to which it remains a viable therapeutic target remains unknown. To address this question we exposed immortalized (p53/pRb-deficient), untransformed human astrocytes to the mutant IDH1 inhibitor AGI-5198 prior to, concomitant with, or at intervals after, introduction of transforming mutant IDH1, then measured effects on 2-HG levels, histone methylation (H3K4me3, H3K9me2, H3K9me3 or H3K27me3) and growth in soft-agar. Addition of AGI-5198 prior to, or concomitant with, introduction of mutant IDH1 blocked all mutant IDH1-driven changes including cellular transformation. Addition at time intervals as short as 4 days following introduction of mutant IDH1 also suppressed 2-HG levels, but had minimal effects on histone methylation, and lost the ability to suppress clonogenicity in a time-dependent manner. Furthermore, in two different models of mutant IDH1-driven gliomagenesis, AGI-5198 exposures that abolished production of 2-HG also failed to decrease histone methylation, adherent cell growth, or anchorage-independent growth in soft-agar over a prolonged period. These studies show although mutant IDH1 expression drives gliomagenesis, mutant IDH1 itself rapidly converts from driver to passenger.
\end{abstract}

Implications-Agents that target mutant IDH may be effective for a narrow time and may require further optimization or additional therapeutics in glioma.

Corresponding Author: Russell O. Pieper. UCSF Helen Diller Family Cancer Research Building, 1450 S. 3rd St, Rm HD287, Box 0520, San Francisco, CA 94158-9001 USA, Tel: (415) 502-7132; Fax: (415) 502-6779; rpieper@cc.ucsf.edu.

There are no relationships that could be construed as resulting in an actual, potential, or perceived conflict of interest with regard to this manuscript. 


\section{Keywords}

mutant IDH1; mutant IDH1 inhibitor; 2-HG; glioma

\section{Introduction}

Mutations in isocitrate dehydrogenase 1 and 2 (IDH1 and IDH2) have been noted in several types of cancers including acute myeloid leukemia $(1,2)$, non-epithelial melanoma (3), and glioma $(4,5)$. IDH1 mutations, however, are among the most common alterations in glioma, and nearly $70 \%$ of lower grade gliomas contain heterozygous R132H mutations at the enzyme's active site (6). R132H IDH1 mutation in glioma results in the creation of a mutant IDH1 protein which dimerizes with the wild-type (WT) IDH1 $(7,8)$. Detailed biochemical studies have shown that while cytoplasmic WT IDH1 readily converts isocitrate to aketoglutarate (a-KG)(9), the WT/mutant dimer has a relatively higher affinity for a-KG, and displays a neomorphic activity that converts a-KG to 2-hydroxyglutarate (2-HG)(9). 2-HG accumulates in IDH1-mutant cells and competes with a-KG for binding to a wide variety of a-KG-dependent enzymes (10). The end result of 2-HG accumulation is the inhibition of multiple enzymes involved in the control of cytosine DNA methylation and histone methylation (11-14), wide-spread changes in gene expression (15), and a strong association with cellular transformation $(16,17)$. In lower-grade glioma, an analysis of clonality also suggests that IDH1 mutation is among the earliest events, and occurs before TP53 mutation in astrocytomas or loss of $1 \mathrm{p} / 19 \mathrm{q}$ in astrocytomas and oligodendrogliomas (18-21). Based on these findings a series of mutant IDH1 inhibitors have been developed and are in clinical testing $(22,23)$.

Despite the potential promise of mutant IDH1 inhibitors, the evidence that inhibition of mutant IDH1 can suppress the growth of mutant IDH1-driven gliomas is limited. Early studies showed that the histone and DNA hypermethylation, as well as the transformation of leukemic cells induced by mutant IDH1 expression, could be readily reversed by incubation with the selective mutant IDH1 inhibitor AGI-5198 $(24,25)$. Subsequent studies in a single mutant IDH1-containing patient-derived glioma xenograft suggested that exposures to the same IDH1-specific inhibitor suppressed 2-HG levels, reversed at least some of the mutant IDH1-driven epigenetic alterations, and also suppressed clonogenicity (16). Subsequent studies, however, using other mutant IDH1-containing cells reached more mixed results (26, 27), and the most recent studies with a series of xenografts derived primarily from patients with recurrent, mutant IDH-containing gliomas suggest that mutant IDH1 inhibitors have modest growth-suppressing effects (28). Although most of these studies used the same mutant IDH1 inhibitor (AGI-5198), all of the studies used cells in which mutant IDH1 was expressed in cells transformed by other means, or cells in which mutant IDH1 expression appeared to play a secondary role in the transformation process. The validity of mutant IDH1 as a therapeutic target, and in particular in glioma, therefore remains an open question.

We previously described two distinct isogenic models of gliomagenesis in which the transformation of p53/pRb-deficient astrocytes was driven exclusively by mutant IDH1 expression $(29,30)$. In the first system expression of mutant IDH1 in astrocytes made p53/ 
pRb-deficient by expression of the HPV E6 and E7 proteins and immortalized by expression of exogenous hTERT led to direct cellular transformation (29). In the second, the expression of mutant IDH1 in telomerase-negative p53/pRb-deficient astrocytes led to the gradual emergence of a telomerase-positive transformed population of cells that grew in soft agar and in animals (30). Because mutant IDH1 is the sole initiating factor in driving the gliomagenesis process in both these systems, both models have been extensively used to study mutant IDH1 biology $(15,31)$, and both represent optimal systems to address questions related to the role of mutant IDH1 and mutant IDH1-driven processes in initiating and maintaining the transformation process. Using these two systems we here show that the mutant IDH1-driven events that result in cellular transformation occur rapidly (within 3 days) following introduction of the oncogenic insult, and that while these events can be blocked by prior exposure to a selective mutant IDH1 inhibitor, the events driving cellular transformation rapidly become irreversible, making selective mutant IDH inhibitors of marginal use in suppressing the growth and clonogenicity of the mutant IDH1-driven tumors in the systems used.

\section{Materials and Methods}

\section{Cell lines and reagents}

Normal human astrocytes (NHAs) expressing E6, E7, hTERT, and either wild-type (WT) or mutant IDH1 were created and cultured as previously described (32-34). In this series, only cells expressing E6, E7, hTERT, and mutant IDH1 were transformed as defined by their ability to grow in soft agar and to form tumors in animals. The same NHAs expressing E6, E7, and either WT or mutant IDH1, then assayed before they reached the end of their normal lifespan (pre-crisis) or after they emerged from crisis as transformed cells (post-crisis) were also created and cultured as previously described (30). In this series, only the post-crisis cells expressing E6, E7, and mutant IDH1 were transformed as defined by their ability to grow in soft agar and to form tumors in animals. Transient expression of mutant (R132H) IDH1 in NHAs expressing E6, E7, and hTERT was performed as described previously (29). Briefly, multiple wells containing $1 \times 10^{5}$ cells were infected in 6-well format by lentivirus encoding GFP and either mutant IDH1 or a blank construct in the presence of polybrene (Sigma, St. Louis, MO). Successful lentiviral transduction was assayed by fluorescence microscopy and western blotting using a mutant IDH1 specific antibody at the indicated time points. The selective mutant IDH1 inhibitor AGI-5198 (Calbiochem, San Diego, CA) was dissolved in DMSO to obtain a $20 \mathrm{mM}$ stock solution.

\section{Cell proliferation and soft-agar assay}

Cell proliferation of control and AGI-5198-treated cells was determined every 7 days for up to 28 days by trypan blue exclusion counting as described previously (32). Soft-agar assays were performed in 6-well plates where the bottom layer contained complete DMEM media (10\% FBS and $1 \%$ penicillin/streptomycin) in $0.7 \%$ agar, the middle layer consisted of complete DMEM media with 5000 cells in $0.35 \%$ agar, and the top layer was made of complete DMEM media in 0.7\% agar. Cells were pre-treated with vehicle or AGI-5198 for $72 \mathrm{hrs}$ before being embedded in soft-agar, and all soft-agar layers contained either vehicle 
or AGI-5198. Colonies were fixed and stained with $0.005 \%$ crystal violet after 28 days, and the colonies counted.

\section{Protein extraction and immunoblotting}

Whole-cell protein lysates were prepared using RIPA Lysis and Extraction Buffer (Thermo Scientific, Rockford, IL) supplemented with PhosSTOP phosphatase and cOmplete, Mini, protease inhibitor cocktail (Roche Diagnostics, Indianapolis, IN). Nuclear proteins were extracted using Subcellular Protein Fractionation Kit (Thermo Scientific) according to the manufacturer's instructions. Protein lysates were quantified with DC Protein Assay (BioRad Laboratories, Hercules, CA). Equal amounts of whole-cell (30 $\mu \mathrm{g})$ or nuclear protein extracts $(20 \mu \mathrm{g})$ were electrophoresed and transferred onto a PVDF membrane (Bio-Rad Laboratories) under standard conditions. The following primary antibodies were used: IDH1 R132H (DIA-H09, Dianova GmbH), H3K4me3 (\#39159), H3K9me2 (\#39683), H3K9me3 (\#39161), H3K27me3 (\#39155; all from Active Motif, Carlsbad, CA), Histone H3 (ab1791, Abcam, Cambridge, MA), GAPDH (\#14C10, Cell Signaling Technology, Danvers, MA). The corresponding secondary antibodies anti-mouse (sc-2005) and anti-rabbit (sc-2004) IgG-horseradish peroxidase (HRP) were from Santa Cruz Biotechnology (Dallas, TX). Antibody binding was detected using Amersham ECL Western Blotting Detection Reagent (GE Healthcare Life Sciences, Pittsburgh, PA).

\section{Measurements of intracellular 2-HG levels}

Metabolites were extracted after treatment with either drug vehicle or AGI-5198 at the indicated time points from approximately $3 \times 10^{7}$ cells in each sample by dual phase extraction as described previously (35). 1H-MRS spectra (1D water presaturation ZGPR sequence, $90^{\circ}$ flip angle, $3 \mathrm{~s} \mathrm{TR}, 256$ acquisitions) were acquired using a $500 \mathrm{MHz}$ Avance spectrometer (Bruker BioSpin) equipped with a Triple Resonance CryoProbe. Metabolites were quantified by normalizing to a trimethylsilyl propanoic acid reference of known concentration and correcting for saturation and Nuclear Overhauser effect.

\section{Statistical analyses}

Data are presented as mean \pm SEMs of 3 independent experiments. Statistical analyses were carried out using a two-tailed Student's t-test assuming equal variances. When multiple groups were evaluated, the one-way ANOVA test with post hoc Turkey-Kramer multiple comparisons test was used. $\mathrm{P}<0.05$ was considered statistically significant.

\section{Results}

\section{Mutant IDH1 rapidly converts from driver to passenger in the gliomagenesis process}

To begin to address the potential of mutant IDH1 as a therapeutic target in glioma, we first verified that mutant IDH1 expression resulted in production of the 2-HG oncometabolite in our gliomagenesis models, and that treatment with a mutant IDH1 inhibitor resulted in suppression of 2-HG levels. While cell groups expressing only WT IDH1 exhibited only background levels of 2-HG (not shown), all cells engineered to express mutant IDH1 (IDH1 mutant pre- and post-crisis cells, and hTERT IDH1 mutant cells) contained measurable levels of 2-HG (Fig 1) ranging between 6 and $14 \mathrm{fmol} / \mathrm{cell}$. In each case exposure of cells to 
the mutant IDH1-inhibitor AGI-5198 led to >98\% suppression of 2-HG levels within 3 days of exposure to 1 or $10 \mu \mathrm{M}$ of the compound. Continuous exposure of the cells to AGI-5198 resulted in suppression of 2-HG levels as long as the cells were followed (up to 28 days post initiation of drug exposure).

To assess the contribution of mutant IDH1 to the transformation of glioma cells, and the need for continued mutant IDH1 expression, we first transiently infected E6E7hTERT immortalized astrocytes with a lentiviral construct encoding mutant IDH1 and a fluorescent marker, then verified the effect of mutant IDH1 expression on histone modification and the growth of cells in soft agar (schematic, Fig 2A, line 9). Greater than $90 \%$ of the target cells were infected (based on fluorescence microscopy) and, as shown in Fig 2B, cells transfected with the construct encoding mutant IDH1 had significantly higher levels of histone modifications (H3K4me3, H3K9me2, H3K9me3, H3K27me3) known to be driven by mutant IDH1 expression (lane 9)(15) than the same cells 4 days after transiently transfection with a blank vector (lane 1). These cells also formed colonies in soft agar consistent with the ability of mutant IDH1 to drive transformation (group 9 vs group 1, Fig 2C). Persistent exposure of the cells to a 2-HG-suppressing concentration of AGI-5198 $(1 \mu \mathrm{M})$ beginning three days prior to introduction of the mutant IDH1 construct completely blocked the increased levels of mutant IDH1-driven histone modifications (measured 4 days after mutant IDH1 introduction) (lane 2) as well as the ability of mutant IDH1 to drive cellular transformation and the growth of cells in soft agar. Persistent exposure of cells to AGI-5198 beginning at the time of introduction of mutant IDH1 similarly blocked the increased levels of mutant IDH1-driven histone modifications (again 4 days after introduction of mutant IDH1) (lane 3) and the growth of cells in soft agar, showing that mutant IDH1 is an inhibitable driver of transformation in these cells.

To address whether mutant IDH1 expression was required to maintain the transformed state, cells infected with the mutant IDH1-encoding construct were continuously exposed to $1 \mathrm{uM}$ AGI-5198 beginning 4 to 21 days after introduction of the oncogenic mutant IDH1 lesion, then assayed for histone modifications and clonogenicity 3-4 days after initiation of drug treatment. As shown in Fig 2B, delaying onset of mutant IDH1 inhibition for even 4 days greatly diminished the ability of the drug to suppress levels of the mutant IDH1-driven histone modifications measured (lane 4 vs lane 2), as well as the ability of the drug to suppress growth in soft agar (Fig $2 \mathrm{C}$ group 4 vs group 2). Increasing the time between mutant IDH1 introduction and mutant IDH1 inhibitor treatment also led to a time-dependent increase in histone modifications (Fig 2B lanes 5-7) and clonogenicity (Fig 2C groups 5-7). Furthermore, although AGI-5198 could suppress clonogenicity if applied before oncogenic insult, the removal of AGI-5198 in the presence of the mutant IDH1 oncogenic insult led within four days to the generation of cells that again regained alterations in histone methylation (Fig 2B lane 8 vs lane 2) and the ability to grow in soft agar (Fig 2C group 8 vs group 2). Collectively these results suggest that oncogenic effects of mutant IDH1 in this system are only marginally reversible, and that this window of opportunity is essentially lost within even 3 days of oncogenic insult. 


\section{Mutant IDH1 inhibition has minimal effect on the growth, histone modifications, and clonogencity of IDH1 mutant-driven glioma cells}

To more fully determine if mutant IDH1 represents a reasonable pharmacologic target in IDH1 mutant-driven transformed cells, we expanded our studies to include a second IDH1transformed glioma cell line and a broader and longer range of drug exposures. As shown in Fig 3, concentrations of AGI-5198 that were capable of suppressing levels of 2-HG by $>98 \%$ did not alter the growth of non-transformed E6E7hTERT cells expressing WT IDH1 (A), or of E6E7hTERT cells transformed by expression of mutant H-Ras (B). Consistent with data in Fig 2, these drug exposures also had no effect on the growth of E6E7hTERT transformed by expression of mutant IDH1 (C), even after up to 28 days of continuous drug exposure. Similar studies performed in a second independent mutant IDH1-transformed glioma cell lines yielded identical results. Specifically, concentrations of AGI-5198 that were capable of suppressing levels of 2-HG by $>98 \%$ did not alter the growth of non-transformed E6E7 cells expressing WT IDH1 (D), or of E6E7 cells expressing mutant IDH1 prior to (pre-crisis) (E) or after (post-crisis) (F) their transformation by mutant IDH1 expression, even after up to 28 days of continuous drug exposure.

The lack of effect of AGI-5198 on the growth of these cells was mirrored by an inability of these drug exposures to suppress mutant IDH1-driven increases in histone modifications, which remained elevated in the drug-treated E6E7 mutant IDH1 pre- and post-crisis cells (Fig 4 panels B and C) relative to those in the control (first lane, panels $4 \mathrm{~B}$ and $\mathrm{C}$ ) or AGI-5198-treated (Fig 4A) E6E7 WT IDH1 cells, and in the drug-treated E6E7hTERT cells expressing mutant IDH1 (Fig 4E) relative to those in the control (first lane, panel 4E) or AGI-5198-treated E6E7hTERT WT IDH1 cells (Fig 4D), even after 28 days of drug exposure.

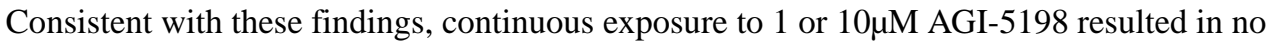
significant change in clonogenicity in non-transformed E6E7hTERT cells expressing WT IDH1 (Fig 5A) or in E6E7hTERT cells transformed by mutant V12H-Ras (Fig 5B). The same drug exposures caused only a modest decrease in clonogenicity in cells transformed E6E7hTERT cells expressing mutant IDH1 (Fig 5C) and in E6E7 cells transformed by mutant IDH1 (post-crisis cells; Fig 5D), and only at drug exposures far beyond those needed for suppression of 2-HG levels. These findings as a whole show that although mutant IDH1 expression drives changes in histone methylation and cellular transformation, mutant IDH1 remains a viable therapeutic target for only a brief period of time.

\section{Discussion}

Because IDH1 mutation is among the earliest and most common alterations in lower grade glioma, there is considerable enthusiasm for the possibility that the mutant IDH1 protein may represent a viable therapeutic target in the treatment of lower-grade glioma. Initial results in studies using cells expressing exogenous mutant IDH1, and in cells from mutant IDH1 containing patient-derived xenografts, however, have yielded mixed results, in part because mutant IDH1 may not be a driver, or the sole driver, of the tumorigenic process in the cell models used. To more clearly address the fundamental question of whether mutant IDH1 represents a reasonable therapeutic target in glioma, we assessed the ability of a 
widely used and selective mutant IDH1 inhibitor to block growth, histone methylation, and clonogenicity in two cellular models in which mutant IDH1 is the sole driver of the transformed phenotype $(29,30)$. The results of these studies clearly show that while mutant IDH1 can drive gliomagenesis, its viability as a therapeutic target appears limited by its rapid transition from a driver to a passenger in the transformation process (Fig 6).

The present studies confirm that mutant IDH1 is a driver of gliomagenesis and further show that mutant IDH1-driven gliomagenesis can occur more rapidly than previously appreciated. Previous studies suggested that changes in histone modification and DNA cytosine methylation driven by mutant IDH1 expression in glioma cells accumulate gradually over time (15), implying that the transformation process driven by mutant IDH1 may be similarly gradual. In the immortalized cells in the present studies, however, changes in histone modification occurred within 4 days of mutant IDH1 introduction, and these modifications remained essentially unchanged 4 weeks after introduction of the oncogenic insult. Furthermore, the mutant IDH1-transformed immortalized astrocytes exhibited only modest increases in global $\mathrm{CpG}$ methylation and not a frank gCIMP phenotype when analyzed several weeks after creation (data not shown). The approach used, however, allowed us to show that even 4 days of unopposed mutant IDH1 expression were sufficient to activate processes that led to cellular transformation. Although the present studies do not address the linkage between changes in histone modification, DNA cytosine methylation, and transformation, changes in histone methylation appear to be at least temporally linked to cellular transformation, while the gCIMP phenotype appears less important. Furthermore, if additional changes in histone modification occurred beyond the last time point studied, these changes appear not to be required for transformation. Based on the data generated in this system, the temporal window of opportunity to block mutant IDH-induced transformative changes therefore appears very narrow.

The present results also show that continued mutant IDH1 protein activity does not appear to be required for maintenance of the glioma phenotype. Although mutant IDH1 expression resulted in cellular transformation, and although pre-treatment of cells with an IDH1 inhibitor blocked mutant IDH1-mediated transformation, effective mutant IDH1 inhibition initiated after oncogenic insult failed to reverse mutant IDH1-mediated changes in histone modification or transformation. Consistent with these results, even one month exposure to a mutant IDH1 inhibitor had minimal effects on mutant IDH1-induced histone modifications, and on the growth and clonogenicity of two different models of IDH1 mutant-driven gliomagenesis. The behavior of mutant IDH1 in this regard differs from that of other oncogenic insults such as c-myc and $\mathrm{H}$-ras, which in general require sustained activation to maintain the tumorigenic phenotype (35-39). Although the current studies do not eliminate the possibility that presence of mutant IDH1, even in an AGI-5198-inhibited form, is important in the maintenance of tumor cell growth $(8,40)$, the dispensability of mutant IDH1 activity for the continued growth and clonogenicity of mutant IDH1-driven glioma cells suggests that inhibition of mutant IDH1 activity may represent a sub-optimal therapeutic approach in glioma

The observation that mutant IDH1 activity is important in initiating, but not maintaining, the gliomagenic state may have important clinical implications. If already established mutant 
IDH1 tumors are no longer dependent on mutant IDH1 activity, the utility of mutant IDH1selective inhibitors would appear to be limited. IDH1 mutation, however, appears to temporally precede genetic alterations associated with resolution of telomeric dysfunction such as ATRX (18-21). Furthermore, in the telomere-deficient model used here, the resolution of telomeric dysfunction appears to be a temporal bottleneck in the mutant IDH1driven transformation of telomere-negative cells, and this resolution can take several months in culture (30). It therefore remains possible that mutant IDH1 inhibitors may limit the ability of cells to resolve telomeric dysfunction and therefore have a role in controlling recurrence. Alternatively, changes in metabolism induced by expression of mutant IDH1 and the shunting of a-KG to 2-HG may make cells collaterally sensitive to other metabolically targeted therapies (28) which may or may not require inhibition of mutant IDH1. The present studies nonetheless show that while IDH1 mutation is an important driver of gliomagenesis, its utility as a direct therapeutic target may be less than optimal.

\section{Acknowledgments}

Grant Support: This work was supported in part by National Institutes of Health Grants CA172845-03 (to SMR and ROP), CA171610-03 (to ROP), the Loglio Research Project (to SMR, and ROP), and The Kristian Gerhard Jebsen Foundation and The Norwegian Cancer Society (to T-CAJ and RB)

\section{References}

1. Paschka P, Schlenk RF, Gaidzik VI, Habdank M, Krönke J, Bullinger L. IDH1 and IDH2 mutations are frequent genetic alterations in acute myeloid leukemia and confer adverse prognosis in cytogenetically normal acute myeloid leukemia with NPM1 mutation without FLT3 internal tandem duplication. J Clin Oncol. 2010; 28:3636-43. [PubMed: 20567020]

2. Mardis ER, Ding L, Dooling DJ, Larson DE, McLellan, Chen K, et al. Recurring mutations found by sequencing an acute myeloid leukemia genome. N Eng J Med. 2009; 361:1058-61.

3. Shibata T, Kokubu A, Miyamoto M, Sasajima Y, Yamazaki N. Mutant IDH1 confers an in vivo growth advantage in a melanoma cell line with BRAF mutation. Am J Path. 2011; 178:1395-1402. [PubMed: 21356389]

4. Parsons DW, Jones S, Zhang X, Lin JC, Leary RJ, Angenendt P. An integrated genomic analysis of human glioblastoma multiforme. Science. 2008; 321:1807-12. [PubMed: 18772396]

5. Yan H, Parsons DW, Jin G, McLendon R, Rasheed BA, Yuan W, et al. IDH1 and IDH2 mutations in gliomas. N Eng J Med. 2009; 360:765-73.

6. Balss J, Meyer J, Mueller W, Korchunov A, Hartmann C, et al. Analysis of the IDH1 codon 132 mutation in brain tumors. Acta Neuropathol. 2008; 116:597-602. [PubMed: 18985363]

7. Dang L, White DW, Gross S, Bennett BD, Bittinger MA, Driggers EM, et al. Cancer-associated IDH1 mutations produce 2-hydroxyglutarate. Nature. 2009; 462:739-47. [PubMed: 19935646]

8. Jin G, Reitman ZJ, Duncan CG, Spasojevic I, Gooden DM, Rasheed BA, et al. Disruption of wildtype IDH1 suppresses D-2-hydroxyglutarate production in IDH1-mutated gliomas. Cancer Res. 2013; 73:496-501. [PubMed: 23204232]

9. Pietrak B, Zhao H, Qi H, Quinn C, Gao E, Boyer JG, et al. A tale of two subunits: How the neomorphic R132H mutation enhances production of aHG. Biochemistry. 2011; 50:4804-12. [PubMed: 21524095]

10. Xu W, Yang H, Liu Y, Yang Y, Wang P, Kim S-H, et al. Oncometabolite 2-hydroxyglutarate is a competitive inhibitor of a-ketoglutarate-dependent dioxygenases. Cancer Cell. 2011; 19:17-30. [PubMed: 21251613]

11. Figueroa ME, Abdel-Wahab O, Lu C, Ward PS, Patel J, Shih A, et al. Leukemic IDH1 and IDH2 mutations result in a hypermethylation phenotype, disrupt TET2 function, and impair hematopoetic differentiation. Cancer Cell. 2010; 18:553-67. [PubMed: 21130701] 
12. Sasaki M, Knobbe CB, Munger JC, Lind EF, Brenner D, Brustle A, et al. IDH1 (R132H) mutation increase murine haematopoietic progenitors and alters epigenetics. Nature. 2012; 488:656-9. [PubMed: 22763442]

13. Koch CM, Andrews RM, Flicek P, Dillon SC, Karoaz U, Clelland GK, et al. The landscape of histone modifications across $1 \%$ of the human genome in five human cell lines. Genome Res. 2007; 176:691-707.

14. Barski A, Cuddapah S, Cui K, Roh TY, Schones DE, Wang Z, et al. High-resolution profiling of histone methylations in the human genome. Cell. 2007; 1294:823-37.

15. Lu C, Ward PS, Kapoor GS, Rohle D, Turcan S, Abdel-Wahab O, et al. IDH mutation impairs histone demethylation and results in a block to cell differentiation. Nature. 2012; 483:474-8. [PubMed: 22343901]

16. Rohle D, Popovici-Muller J, Palaskas N, Turcan S, Grommes C, Campos C, et al. An inhibitor of mutant IDH1 delays growth and promotes differentiation of glioma cells. Science. 2013; 340:62630. [PubMed: 23558169]

17. Chaumiel MM, Larson PEZ, Yoshihara HAI, Danforth OM, Vigneron DB, Nelson SJ, et al. Noninvasive in vivo assessment of IDH1 mutational status in glioma. Nature Comm. 2013; 4:2429-41.

18. Lai A, Kharbanda S, Pope WB, Tran A, Solis OE, Peale F, et al. Evidence for Sequenced Molecular Evolution of IDH1 Mutant Glioblastoma From a Distinct Cell of Origin. J Clin Oncol. 2011; 29:4482-90. [PubMed: 22025148]

19. Watanabe T, Nobusawa S, Kleihues P, Ohgaki H. IDH1 mutations are early events in the development of astrocytomas and oligodendrogliomas. Am J Pathol. 2009; 174:1149-53. [PubMed: 19246647]

20. Johnson BE, Mazor T, Hong C, Barnes M, Aihara K, McLean CY, et al. Mutational analysis reveals the origin and therapy-driven evolution of recurrent glioma. Science. 2014; 343:189-93. [PubMed: 24336570]

21. Kannan K, Inagaki A, Silber J, Gorovets D, Zhang J, Kastenhuber ER, et al. Whole-exome sequencing identifies ATRX mutation as a key molecular determinant in lower-grade glioma. Oncotarget. 2012; 3:1194-203. [PubMed: 23104868]

22. Dang L, Yen K, Attar EC. IDH mutations in cancer and progress toward development of targeted therapeutics. Ann Oncol. 2016; 27:599-608. [PubMed: 27005468]

23. Clark O, Yen K, Mellinghoff IK. Molecular Pathways: Isocitrate Dehydrogenase Mutations in Cancer. Clin Cancer Res. 2016; 22:1837-42. [PubMed: 26819452]

24. Losman JA, Looper RE, Koivunen P, Lee S, Schneider RK, McMahon C, et al. (R)-2hydroxyglutarate is sufficient to promote leukemogenesis and its effects are reversible. Science. 2013; 339:1621-5. [PubMed: 23393090]

25. Kernytsky A, Wang F, Hansen E, Schalm S, Straley K, Gliser C, et al. IDH2 mutation-induced histone and DNA hypermethylation is progressively reversed by small-molecule inhibition. Blood. 2015; 125:296-303. [PubMed: 25398940]

26. Suijker J, Oosting J, Koornneef A, Struys EA, Salomons GS, Schapp FG, et al. Inhibition of mutant IDH1 decreases D-2-HG levels without affecting tumorigenic properties of chondrosarcoma cell lines. Oncotarget. 2015; 6:12505-19. [PubMed: 25895133]

27. Ma S, Jiang B, Deng W, Gu Z-K, Wu F-Z, Li T, et al. D-2-hydroxyglutarate is essential for maintaining oncogenic property of mutant IDH-containing cancer cells but dispensable for cell growth. Oncotarget. 2015; 6:8606-20. [PubMed: 25825982]

28. Tateishi K, Wakimoto H, Iafrate AJ, Tanaka S, Loebel F, Lelic N, et al. Extreme Vulnerability of IDH1 Mutant Cancers to NAD+ Depletion. Cancer Cell. 2015; 28:773-84. [PubMed: 26678339]

29. Ohba S, Mukherjee J, See WL, Pieper RO. Mutant IDH1-driven cellular transformation increases RAD51-mediated homologous recombination and temozolomide resistance. Cancer Res. 2014; 74:4836-44. [PubMed: 25035396]

30. Ohba S, Mukherjee J, Johannessen T-C, Mancini A, Chow TT, Wood M, et al. Mutant IDH1 expression drives TERT promoter reactivation as part of the cellular transformation process. Neuro Oncol. 2015; 17(suppl 5):v55.doi: 10.1093/neuonc/nov209.01

31. Turcan S, Rohle D, Goenka A, Walsh LA, Fang F, Chan T. IDH1 mutation is sufficient to establish the glioma hypermethylator phenotype. Nature. 2012; 483:479-83. [PubMed: 22343889] 
32. Sonoda Y, Ozawa T, Hirose Y, Aldape KD, McMahon M, Berger MS, et al. Formation of intracranial tumors by genetically modified human astrocytes defines four pathways critical in the development of human anaplastic astrocytoma. Cancer Res. 2001; 61:4956-60. [PubMed: 11431323]

33. Ito M, Ohba S, Gaensler K, Ronen SM, Mukherjee J, Pieper RO. Early Chk1 phosphorylation is driven by temozolomide-induced, DNA double strand break- and mismatch repair-independent DNA damage. PLoS One. 2013; 8(5):e62351. [PubMed: 23667469]

34. Sonoda Y, Ozawa T, Aldape KD, Berger MS, Deen DF, Pieper RO. Akt pathway activation converts anaplastic astrocytoma to glioblastoma multiforme in a human astrocyte model of glioma. Cancer Res. 2001; 61:6674-78. [PubMed: 11559533]

35. Izquierdo-Garcia JL, Cai LM, Chaumeil MM, Eriksson P, Robinson AE, Pieper RO, et al. Metabolic Reprogramming in Mutant IDH1 Glioma Cells. PLoS One. 2015; 10:e118781. [PubMed: 25706986]

36. D’Cruz CM, Gunther EJ, Boxer RB, Hartman JL, Sintasath L, Moody SE, et al. c-MYC induces mammary tumorigenesis by means of a preferred pathway involving spontaneous Kras 2 mutations. Nature Med. 2001; 7:235-9. [PubMed: 11175856]

37. Adhikary S, Eilers M. Transcriptional regulation and transformation by Myc proteins. Nature Reviews Molecular Cell Biology. 2005; 6:635-45. [PubMed: 16064138]

38. Jain M, Arvanitis C, Chu K, Dewey W, Leonhardt E, Trinh M, et al. Sustained loss of a neoplastic phenotype by brief inactivation of MYC. Science. 2002; 297:102-4. [PubMed: 12098700]

39. Chin L, Tam A, Pomerantz J, Wong M, Holash J, Bardeesy N, et al. Essential role for oncogenic Ras in tumour maintenance. Nature. 1999; 400:468-72. [PubMed: 10440378]

40. Jin G, Pirozzi CJ, Chen LH, Lopez GY, Duncan CG, Feng J, et al. Mutant IDH1 is required for IDH1 mutated tumor cell growth. Oncotarget. 2012:774-82. [PubMed: 22885298] 
A

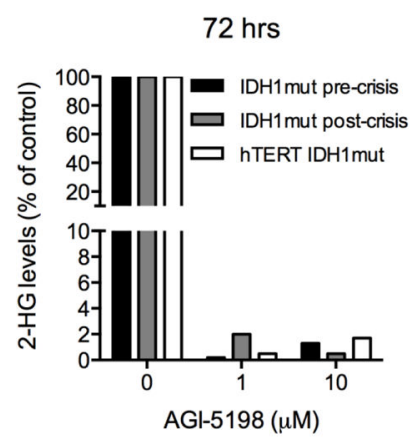

B

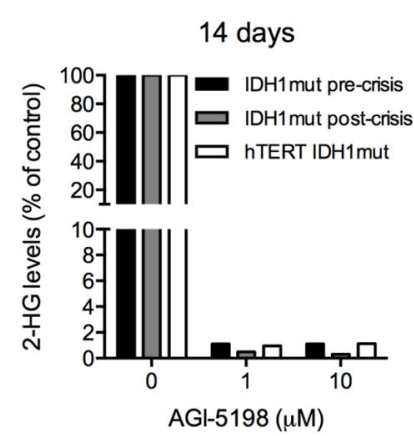

C

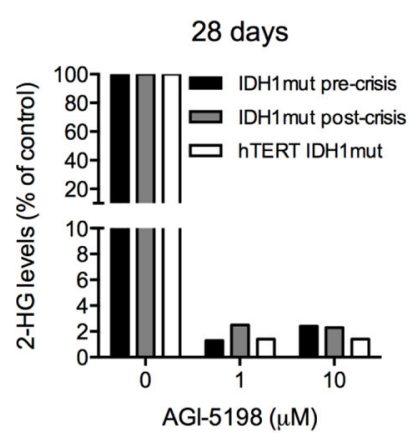

Figure 1.

Continuous treatment with 1 or $10 \mu \mathrm{M}$ AGI-5198 rapidly decreases 2-HG levels in E6/E7 and E6/E7/hTERT cell lines expressing mutant IDH1. Intracellular 2-HG levels were determined by 1H-NMR mass spectroscopy after continuous AGI-5198 exposure for 72 hrs (A) 14 days (B), or 28 days (C). 2-HG levels in AGI-5198 treated cells are expressed as percentage of 2-HG levels in corresponding controls cells receiving vehicle (DMSO) only. 


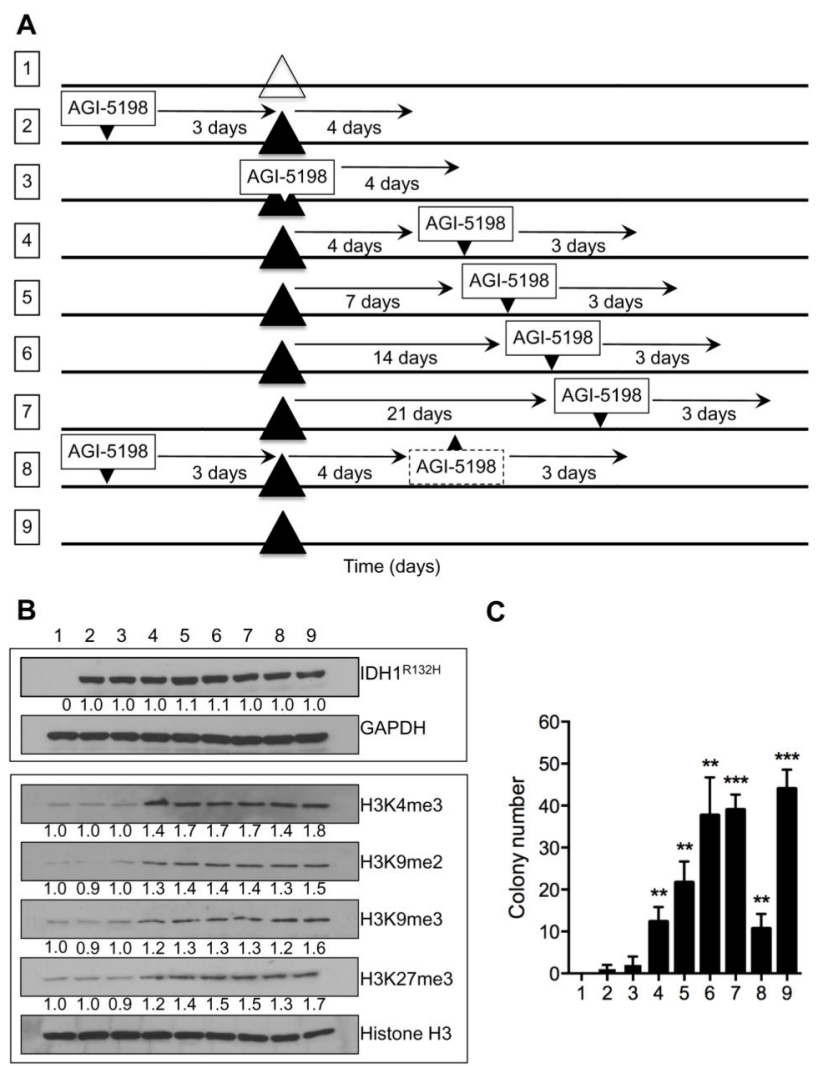

Figure 2.

(A) Schematic overview of the experimental timeline for the different E6/E7/hTERT experimental cell groups (numbered 1-9) following addition (small downward arrowheads) or removal (small upward arrowheads) of $1 \mu \mathrm{M}$ AGI-5198 in temporal relation to the introduction of a blank construct (large open triangle) or a construct encoding mutant IDH1 (large black triangles) and final analysis of histone modifications and clonogenicity. (B) Immunoblotting of mutant IDH1 (upper panel) and various histone $\mathrm{H} 3$ methylation levels (lower panel) in the different E6/E7/hTERT experimental cell groups at the time endpoints indicated in (A). GAPDH and histone $\mathrm{H} 3$ were used as loading controls for whole-cell and nuclear protein lysates, respectively. (C) Soft-agar colony formation as a measure of in vitro cellular transformation in the nine different E6/E7/hTERT cell groups shown in (A).

$* * \mathrm{P}<0.01$. *** $\mathrm{P}<0.001$. 
A

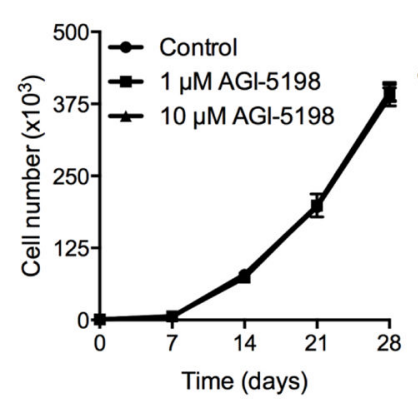

D

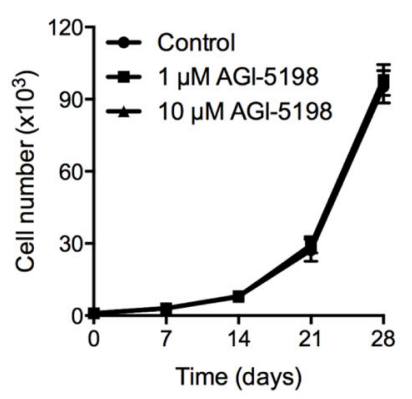

B

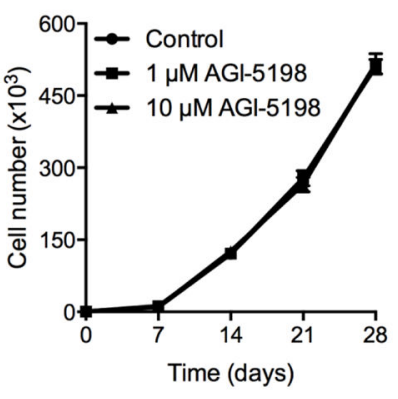

E

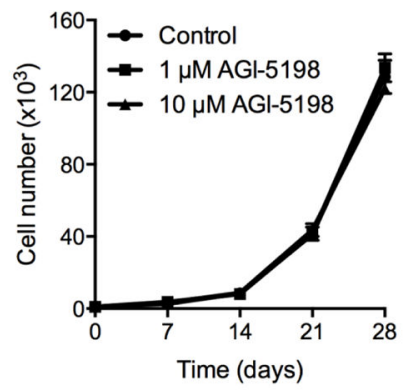

C

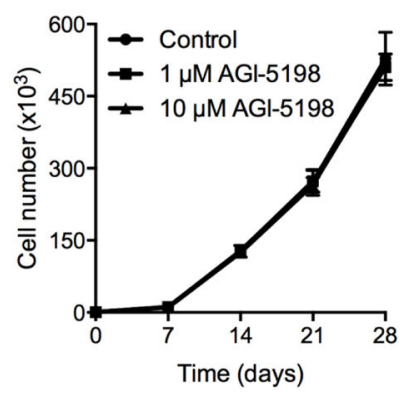

F

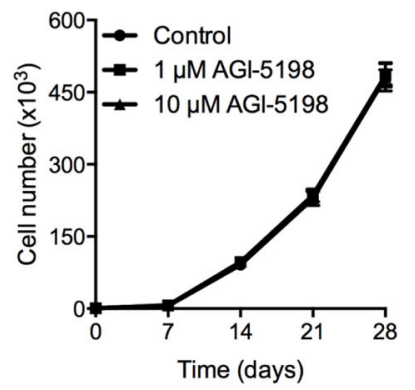

Figure 3.

Growth of the different E6/E7 and E6/E7/hTERT cell lines in response to continuous treatment with drug vehicle (DMSO) or 1 or $10 \mu \mathrm{M}$ AGI-5198 over a time period of 28 days. No reduction in cell proliferation was observed in immortalized E6/E7/hTERT cells expressing wild-type IDH1 (A), E6/E7/hTERT cells transformed independently of mutant IDH1 by mutant V12H-Ras (B), mutant IDH1-driven transformed E6/E7/hTERT cells (C), untransformed E6/E7 cells expressing wild-type IDH1 (D), untransformed E6/E7 cells expressing mutant IDH1 (pre-crisis cells; E), or mutant IDH1-driven transformed E6/E7 cells (post-crisis cells; F). 
A

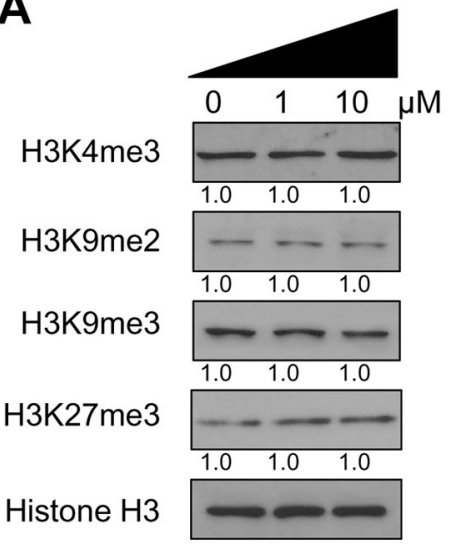

B
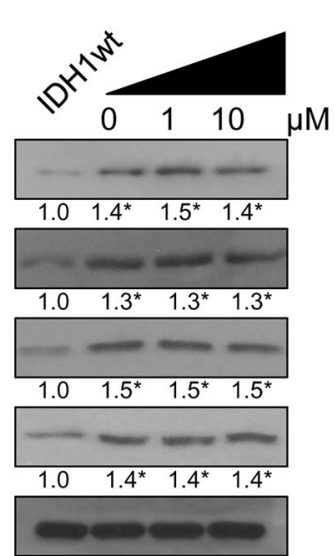

C
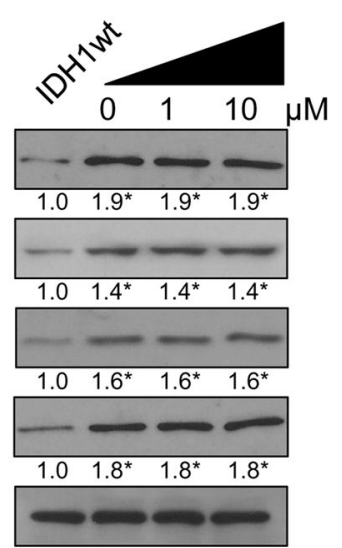

D

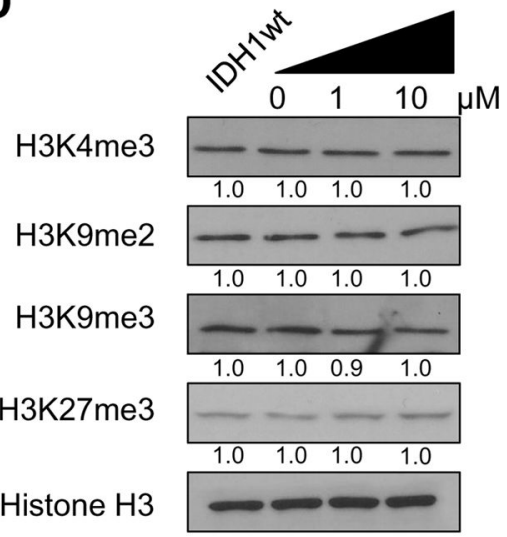

E

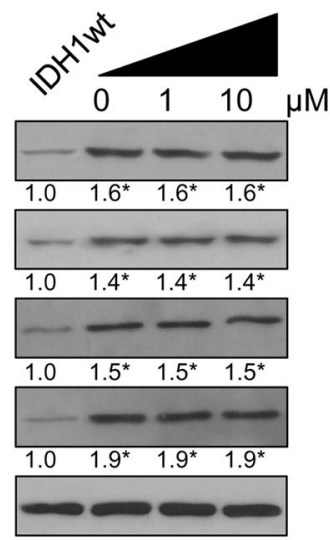

Figure 4.

Immunoblot analysis of lysine di- and trimethylation in histone H3 in E6/E7 and E6/E7/ hTERT cells expressing either WT or mutant IDH1 after 28 days of continuous treatment with vehicle or 1 or $10 \mu \mathrm{M}$ AGI-5198. Untreated E6/E7/IDH1WT cells were used as a control for baseline histone $\mathrm{H} 3$ methylation levels. Total levels of histone H3 were used as loading control. No significant change in H3K4me3, H3K9me2, H3K9me3 or H3K27me3 levels were seen between control and AGI-5198 treated cells in the E6/E7/IDH1WT (A), E6/E7/IDH1mut pre-crisis (B), E6/E7/IDH1mut post-crisis (C), E6/E7/hTERT/IDH1wt (D) or E6/E7/hTERT/IDH1mut (E) cells. $* \mathrm{P}<0.05$. 
A

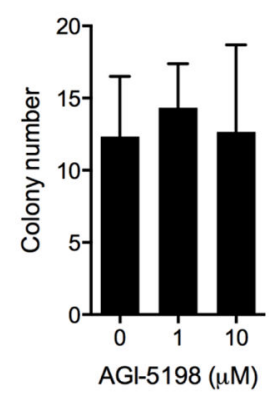

B

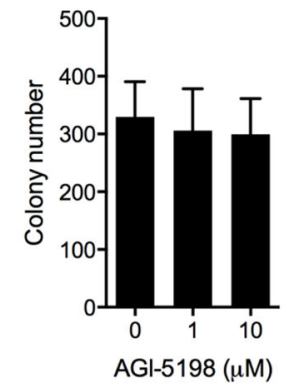

C

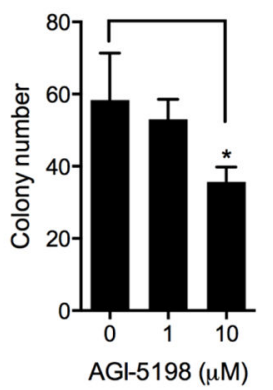

D

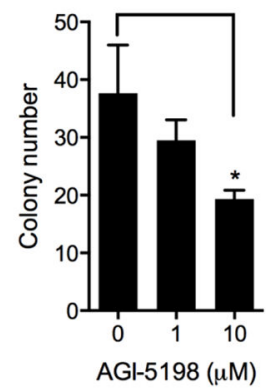

Figure 5.

Colony formation in soft-agar as a measure of anchorage-independent growth in response to 28 day treatment with drug vehicle or 1 or $10 \mu \mathrm{M}$ AGI-5198. No significant reduction in colony formation was noted in E6/E7/hTERT cells expressing wt IDH1 (A) or in cells transformed by expression of mutant V12H-Ras (B). High-dose AGI-5198 (10 $\mu \mathrm{M})$ moderately reduced colony formation in mutant IDH1-driven transformed E6/E7 cells (C) and in mutant IDH1-driven transformed E6/E7 cells (post-crisis, D). ${ }^{*} \mathrm{P}<0.05$. 


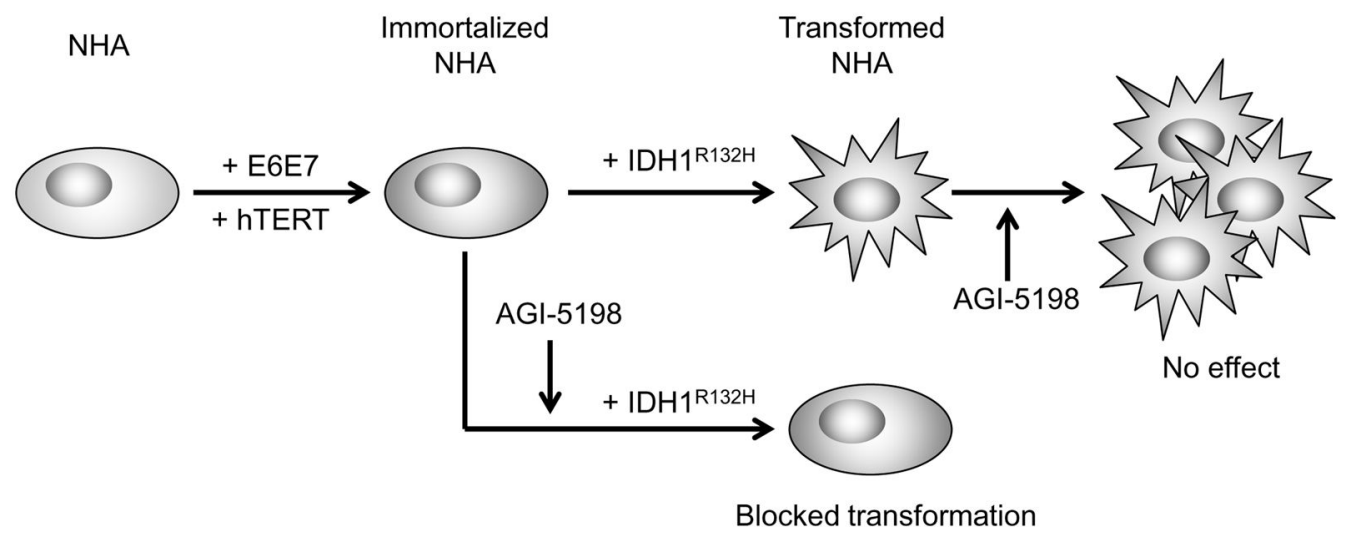

Figure 6.

Potential therapeutic implications for the administration of the mutant IDH1 specific inhibitor AGI-5198. Mutant IDH1-driven transformation of immortalized p53/pRb-deficient human astrocytes can be blocked by AGI-5198 administration prior to or concurrent with the oncogenic insult made by the introduction of mutant IDH1 (1). However, cells already transformed by mutant IDH1 become rapidly insensitive to AGI-5198 (2). This suggests that the temporal window in which effective pharmacological inhibition of mutant IDH1 can achieved appears very limited. 